\title{
Nanoscale
}

\section{Localized self-heating in large arrays of 1D nanostructures}

Received 00th January 20xx, Accepted 00th January 20xx

DOI: $10.1039 / \times 0 \times x 00000 x$

www.rsc.org/

\author{
O. Monereo, ${ }^{\text {a S. Illera, }}{ }^{\text {a A. Varea, }}{ }^{a}$ M. Schmidt, ${ }^{b}$ T. Sauerwald, ${ }^{b}$ A. Schütze, ${ }^{b}$ A. Cirera ${ }^{a}$ and \\ J. D. Prades ${ }^{\mathrm{a}}$
}

One dimensional (1D) nanostructures offer a promising path towards a highly efficient heating and temperature control in integrated microsystems. The so called self-heating effect can be used to modulate the response of solid state gas sensor devices. Efficient self-heating was found to occur at random networks of nanostructured systems with similar power requirements than in highly ordered systems (e.g. individual nanowires, where its thermal efficiency attributed to the small dimensions of the objects). In this work, infrared thermography and Raman spectroscopy were used to map the temperature profiles of films based on random arrangements of carbon nanofibers during self-heating occurrence. Both techniques demonstrate consistently that heating concentrates in small regions, the here-called "hot-spots". Correlating dynamic temperature mapping with electrical measurements, we also observed that these minute hot-spots rule the resistance values observed macroscopically. A physical model of a random network of 1D resistors helped us to explain this observation. The model shows that, for a given random arrangement of 1D nanowires, current spreading though the network ends up defining a set of spots that dominate both the electrical resistance and the power dissipation. Such highly localized heating explains the high power savings observed in larger nanostructured systems. This understanding opens a path to design highly efficient self-heating systems, based on random or pseudo-random distributions of 1D nanostructures.

\section{Introduction}

Electrical measurements in nanostructures can produce remarkable heating effects, even with very low probing signals ${ }^{1-6}$. This so-called self-heating effect was first regarded as an experimental problem in electron devices based on nanowires that should be prevented in all circumstances $^{7-13}$. Later, advancements in low power instrumentation $^{14}$ showed that it was possible to control the selfheating level to the point that it could be used to set the temperature at the nanoscale, without additional heating components ${ }^{15,16}$. In fields requiring temperature control, such as solid state gas sensing, integrating both heating and sensing functionalities in the nanowire itself was a remarkable advantage ${ }^{17}$. More importantly, studies revealed that the self-heating effects in individual nanowires could be used to lower the power consumption figures more than 1000 times compared to state-of-the art microdevices ${ }^{15}$, due to the small dimensions of the volume heated in the nanowire. As a matter of fact, reports demonstrated that operation temperatures of more than $200 \stackrel{\circ}{ } \mathrm{C}$ with less than $20 \mu \mathrm{W}$ were at reach ${ }^{15}$. Moreover, the

\footnotetext{
a. MIND-IN² UB, Department of Electronics, University of Barcelona, 08028, Barcelona, Spain.

b. Lab of Measurement Technology, Department of Mechatronics, Saarland University, 66123, Saarbrucken, Germany.

Electronic Supplementary Information (ESI) available: (i) Text file including detailed description of the experimental procedures and the electrical model used in this work. (ii) MPEG file showing the thermography recording of a heating sequence consisting in five steps: room temperature $\left(\mathrm{RT}, 25^{\circ} \mathrm{C}\right), 100^{\circ} \mathrm{C}$ driven by heater, RT again, $100{ }^{\circ} \mathrm{C}$ driven by self-heating (self-heating nominal temperature, taking the resistance signal as reference ${ }^{34}$ ) and $\mathrm{RT}$ again. The evolution of the temperature distribution histogram, the maximum, average and minimum temperature recorded and its correspondent CNFs film electrical signal. See DOI: $10.1039 / x 0 \times x 00000 x$
}

time needed to thermalize such small masses was explicitly fast, in the range of miliseconds ${ }^{18}$. These timings were comparable to the residence time of many molecules on top of solid surfaces, opening the door to track their interactions and detect them with high specificity ${ }^{19}$. However, all these findings relied on the electrical access to individual nanowires. In spite of the advances of the last years, the fabrication of such devices is still a complex, hardly reproducible, process ${ }^{20}$. Novel methods of top-down nanostructuring with higher yield have been reported ${ }^{21}$, however still major difficulties in term of nanowire size and thermal isolation have to be resolved. That is the reason why this promising approach has just remained as an object of research, with limited applicability ${ }^{22}$.

In the last year, works based on arrays of 1D nanostructures (e.g. nanowires ${ }^{23}$, nanotubes ${ }^{24}$ and nanofibers ${ }^{25}$ ) showed that the requirement of individual nanowires was a misconception. In fact, randomly deposited nanofibers exhibit self-heating effects with performance figures for power dissipation and thermalization time comparable to those of individual nanowires ${ }^{22}$. For example, operation temperatures of $150{ }^{\circ} \mathrm{C}$ with less than $50 \mu \mathrm{W}$ and thermalization times of less than 50 ms were reported ${ }^{26}$ in carbon nanofibers (CNFs) deposited on top of macroscopic interdigitated electrodes (IDE) by techniques as simple as drop casting. Similar results were reported for nearly-random ensembles of metal oxide nanowire connected by means of dielectrophoresis ${ }^{23}$. These figures are close to the ones previously achieved with single nanowire sensors, temperatures of $200{ }^{\circ} \mathrm{C}$ were reached with thermalization times below $10 \mathrm{~ms}$ and power consumption lower than $30 \mu \mathrm{W}^{18}$. Despite the fact that single 1D nanostructures may present better characteristics, the simple techniques required to fabricate non single nanowire sensors facilitate dramatically the applicability of the self-heating effects, reviving again the interest for that approach. 
This context raises a fundamental question on the origin of the high power efficiency of the self-heating effects. First works with individual nanowires proposed that the minute dimensions of the structures lead to remarkably high Joule-dissipated power densities in the nanostructure, even at very low current/voltage levels. Also, the small contacts with the surroundings minimized thermal losses, providing the high thermal efficiency observed ${ }^{15,17}$. These arguments justified the need of individual nanostructures, and suggested that such effects were just unfeasible in large arrangements of nanowires ${ }^{27}$. However, as previously stated, recent experiments show that this is not the case: efficient self-heating can also be achieved in random arrangements of multiple 1D nanostructures ${ }^{23,25}$. This work aims to shed some light on the issue, identifying a broad, general cause for efficient self-heating in nanostructured systems.

\section{Results and discussion}

\section{Temperature mappings}

Infrared thermography. To start, the self-heating occurring in films of $\mathrm{CNFs}^{28}$ was examined by infrared thermography at high spatial resolution ${ }^{29,30}$. The CNFs were deposited on top of a pair of $\mathrm{Pt}$ electrodes. The devices also contained a calibrated heater element for comparison purposes. Before imaging, the temperature reached in self-heating operation was calibrated by monitoring the CNFs film resistance and comparing the values obtained with the heater with those obtained with self-heating. Using this standard procedure $20,25,31-34$, the nominal self-heating temperature was that of the heater at an equivalent resistance drop.

Figure 1a shows the thermal images of the CNFs film at $100{ }^{\circ} \mathrm{C}$ reached with self-heating and heater. Clearly, the self-heating thermal pattern (left picture) is much more inhomogeneous than that observed in the case of the heater (right picture) (see also histogram in Figure $1 \mathrm{~b}$ ). These thermal images clearly suggest that the self-heating is mostly localized in a few "hot spots" that reach temperatures remarkably higher than the film average temperature.

Raman spectroscopy. To confirm this observation and to discard any possible artifact due to the differences in infrared emissivity of the different sample regions, temperature distributions in self-heating operation were also measured with in-situ spatial Raman mappings of the displacement in the $\mathrm{G}$ band of carbonaceous materials ${ }^{35-37}$. Figure 1c shows an example of such Raman maps at different conditions of self-heating, together with temperature distribution histograms. The results are fully equivalent to those observed with direct thermal microscopy: the temperature distribution with selfheating is highly inhomogeneous and characterized by a few spots at remarkably higher temperatures, supporting the idea that selfheating concentrates in hot spots.

In order to understand the origin of the hot-spots, the temperature and the electrical resistance of the CNFs film were monitored, submitting the sample to two equivalent heat pulses, first with the heater and then with self-heating. Figure 2 shows the record of the maximum, average and minimum temperatures of the thermographic image of the CNFs film, synchronized with the electrical resistance measurement. The electrical record during the heater-driven pulse is smooth and comparable to the average temperature evolution. In contrast, the electrical record with selfheating exhibits a sudden change in the less than $2.5 \mathrm{~ms}$ (Process A) followed by a slower stabilization process that extends for a few seconds (Process B). Interestingly, the maximum temperature values in self-heating thermal mapping (by definition, the hot-spot) show exactly the same dynamic behavior (see Figure $2 a$ vs Figure $2 b$ ). This strongly suggests that the sudden resistance changes (i.e. process $A$ ) are somehow related to the existence of hot-spots. The first process is covering a large portion of the total thermal emission (in the given example $2 / 3$ of the mean temperature change; see mean temperature signal in Figure 2a during self-heating warm up).

In a network of randomly oriented 1D nanostructures, the intrinsic dispersion of the nanowire properties (e.g. electrical and geometrical), and the specific connections established among them (e.g. in number and topology, but also in contact resistance) will certainly determine the current paths through the network, and thus, the electrical power dissipated at each point. However, due to the branched topology of the structure, predicting which element concentrates most of the power, and thus becomes a hot-spot, is far from being evident. For example, in a simple case of only two resistors in parallel, most of the power would be dissipated in the less resistive one (since, for the same voltage drop, most of the current will flow through that branch). In contrast, in the case of only two resistors in series, most of the power would be dissipated in the most resistive one (since, for one single current path, the largest voltage drop will develop across the biggest resistance).

\section{Resistor network model}

In order to clarify this situation in an arbitrarily complex network, a toy model for a random network of 1D nanostructures was built based on the following assumptions. (i) The network is composed of wire segments distributed between the two electrodes. These segments are randomly placed. Connection nodes appear when two or more wires cross at the same point. Wire segments only connected at one end do not contribute to the electron transport, and are thus not included in the model. All this defines multiple current paths running from one electrode to the other. (ii) The wires are homogeneous nanostructures, i.e. their respective resistance is directly proportional to their length. The temperature dependence is also included being the $i^{\text {th }}$ wire resistance:

$$
\begin{aligned}
R_{W}^{i}= & \rho \frac{L_{i}}{S} R_{C N}\left(T_{i}\right)=R_{0}^{i}\left[1-\alpha_{C N} \Delta T_{i}\right] \\
& R_{0}^{i}
\end{aligned}
$$

where $\rho$ is the material resistivity, $L_{i}$ is the wire length and $S$ the section (uniform thickness for all wires is assumed). The term $R_{C N}\left(T_{i}\right)$ includes linear temperature dependence. 


\section{Nanoscale}

\section{ARTICLE}

(a)



(b)

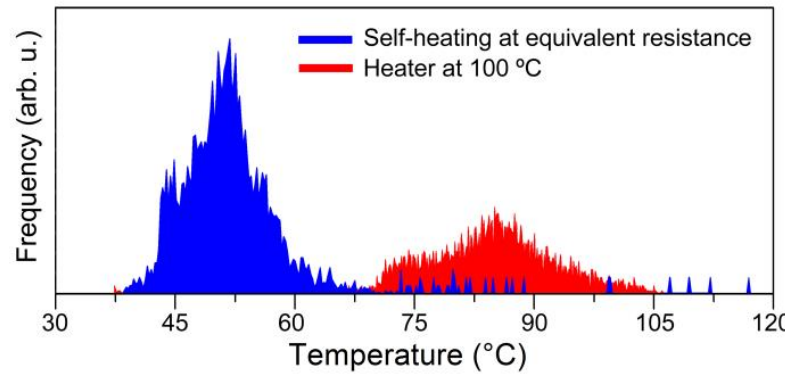

(c)

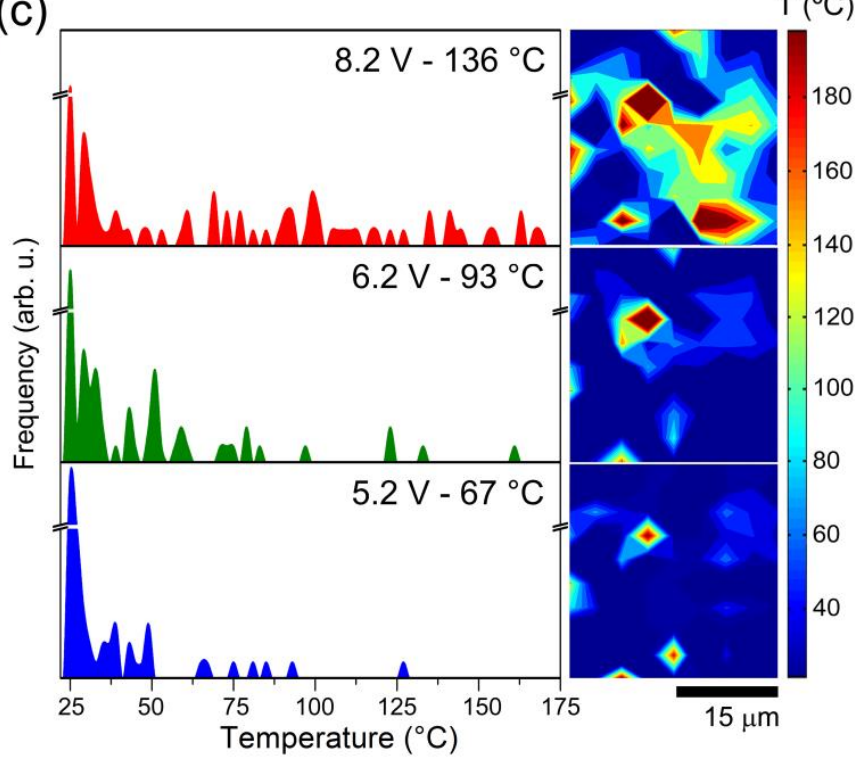

Figure 1. Thermographic image of a CNFs film heated up to a nominal temperature of $100{ }^{\circ} \mathrm{C}$ by means of self-heating (left image) and by an external heater (right image). (b) Histograms of temperature distributions extracted from (a). (c) Raman map-ping of the temperature distribution in a CNF region self-heated at three different nominal temperatures. The left panels show the histograms of the temperature distribution extracted from the corresponding maps (right panels).

(a)

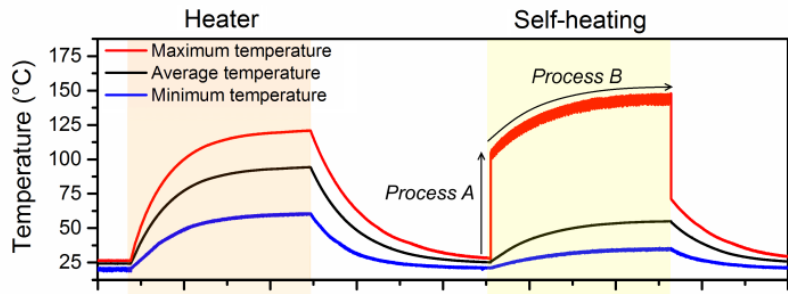

(b)

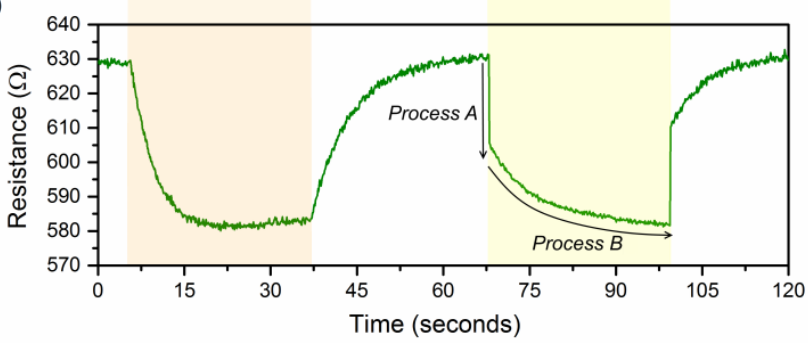

Figure 2. (a) Maximum, average and minimum temperatures of a CNFs film and its corresponding (b) resistance value measured along a heating sequence consisting in five steps: room temperature (RT, $25{ }^{\circ} \mathrm{C}$ ), $100{ }^{\circ} \mathrm{C}$ driven by heater, RT again, $100{ }^{\circ} \mathrm{C}$ driven by selfheating (self-heating nominal temperature, taking the resistance signal as reference ${ }^{34}$ ) and RT again.

This approximation assumes a slowly varying semiconductor resistance for a small temperature range, it does not compromise the generality of the model, and it is valid for the materials used in our experiments 22,38 . None other singularities (such as surface or structural defects) were taken into account in order to maintain the complexity of the system.

The temperature at each wire segment is assumed to be uniform; thus, it is characterized by one single $T_{i}$ value per segment. (iii) The wire-to-wire contacts (i.e. at the connection nodes) are assumed to be proportional to the average resistance of the wires connecting at this node, being the contact resistance for the $j^{\text {th }}$ node:

$$
R_{C}^{j}=\alpha_{R} \sum_{i=1}^{N_{j}} R_{W}^{i}\left(l_{i}, T_{i}\right) / N_{j}
$$

where $N_{j}$ is the number of wires connected at the point $j^{\text {th }}$ and $\alpha_{R}$ is a constant. This approximation accounts for the variability among contacts expected in connections build by the "soft" contact between wires. The $\alpha_{R}$ factor scales the contact resistance value with respect to the wire one, and can be used as weighting factor to directly compare the influence of wires and contacts.

To sum up, the total resistance includes contributions from the wire segments $\left(R_{W}^{j}\right)$ and from the wire-to-wire contacts $\left(R_{C}^{j}\right)$ in random combinations of series and parallel arrangements, which fully mimic the topology of a random array of nanowires lying between a pair of electrodes. Kirchhoff's laws allowed us to determine the current $I_{k}$ flowing through each wire segment and 
crossing each contact resistance under an external voltage $V$ applied across the electrodes. The electrical power dissipated in each $k^{\text {th }}$ element (either wire or contact) can be simply calculated as $P_{k}=I_{k}^{2} \cdot R_{k}$. In order to account for the heating produced, a first order system description is used. Thus, a lineal relationship between the dissipated power and the temperature increment is assumed $\Delta T_{k}=\theta \bullet P_{k}$, where $\theta$ is the thermal resistance and $\Delta T_{k}$ is the temperature increment ${ }^{10}$. This approximation would correspond to the steady solution of a system including heat dissipation and thermal losses (i.e. by conduction, convection, radiation). Since the resistance at each circuit element depends on its temperature, the resistance was updated self-consistently to account for the heating effects.

Figure 3 shows a summary of the results obtained assuming geometry and electrical conditions comparable to the experiments carried out later (resistivity ${ }^{39}$ of $1.4 \times 10^{-3} \Omega \cdot \mathrm{cm}$, wire to wire
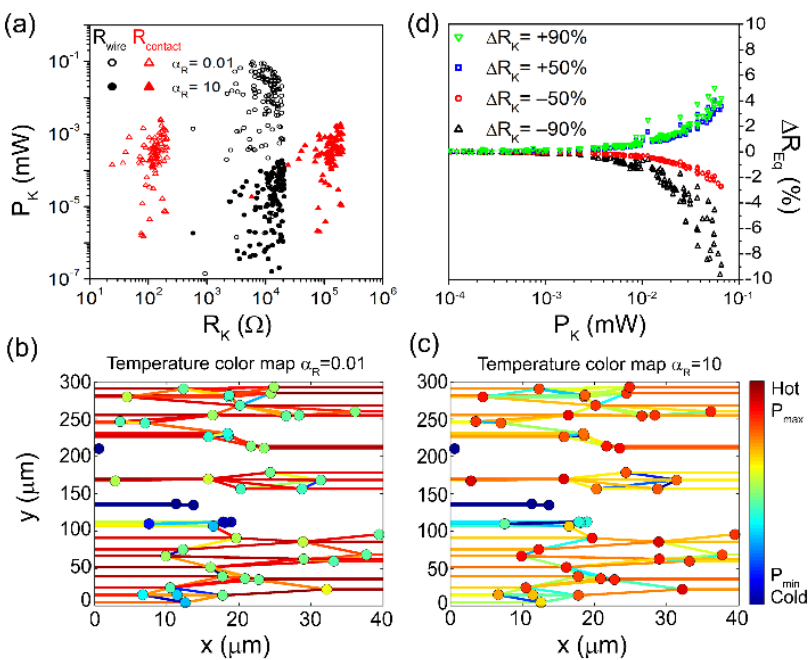

(c)

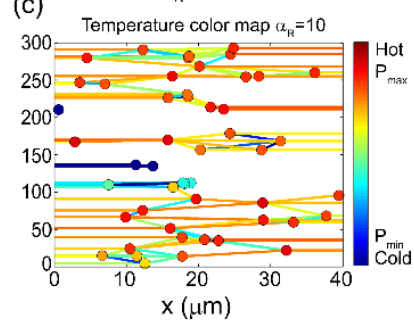

Figure 3. Simulations of power dissipation in random network of resistors. (a) Dissipated power in each element of the system (circles and triangles for contact and wires, respectively) as a function of its resistance for two different values of the scaling parameter $\alpha_{R}$. When the scaling parameter increases, the power is mainly dissipated in the contacts since they become the most resistive elements. (b-c) Power dissipation map, sketching contact nodes as circles and wires segments as lines. Bias voltage was applied from the left to the right edge. The color code corresponds to the power dissipated (i.e. temperature increment) at each element. Each simulation corresponds to a different $\alpha_{R}$ values. (d) Change in the equivalent network resistance $R_{E q}$ as a function of the power $P_{k}$ dissipated in each resistor $R_{k}$ for different resistor variations $\Delta R_{k}$.

length up to $20 \mu \mathrm{m}$, electrode distance of $40 \mu \mathrm{m}$, having approximately $1.2 \times 10^{-2}$ wires $/ \mu \mathrm{m}^{2}$, and bias voltage of $2 \mathrm{~V}$ ). Figure 3a plots the power dissipated $P_{k}$ in each wire and contact, as a function of their corresponding resistance value $R_{k}$, for two different $\alpha_{R}$ values. As expected by construction, the contact resistances increase with $\alpha_{R}$, whereas the wire resistances remain constant. Generally speaking, simulations show that the most dissipative elements (i.e. the ones that could possible reach the highest temperatures) are the most resistive ones. Equivalently, for each $\alpha_{R}$ value, most of the power is dissipated in the most resistive elements: wires for low $\alpha_{R}$ and contacts for high $\alpha_{R}$. Temperature maps of Figure $3 \mathrm{~b}-\mathrm{c}$ provide a visual evidence of the same effect: the most resistive elements (wires for low $a_{R}$-Figure $3 \mathrm{~b}$ - and contacts for high $a_{R}$-Figure 3c-) dissipated most of the power and are thus more likely to reach higher temperatures. Temperature maps also show that this conclusion is true only if the element is properly connected to both electrodes (see blue spots in Figure $3 b-c$ where no dissipation occurs). In conclusion, in random networks of resistors (i) most of the heat dissipation concentrates in the most resistive elements (ii) provided that they are properly connected. If the network is large enough and built in a truly random manner, there will easily be highly resistive elements appropriately connected, and accountable for most of the heat dissipation. All this implies that self-heating in random networks concentrates in some specific points, the previously observed hot-spots, which could possibly correspond to the most resistive regions.

This toy model could also serve to understand the relationship between the temperature evolution observed in the hot-spots and the macroscopic measurements of the electrical resistance. Figure $3 d$ shows the influence of each one of the resistors in the network on the total equivalent resistance value (this is, the value measured experimentally). To that end each resistor value $R_{k}$ was varied, independently, by the same percent $\Delta R_{k}$, and the resulting equivalent resistance of the network $R_{E q}$ was then recalculated. Data was plotted versus the power dissipated $P_{k}$ in the resistor being varied in each case. In a general trend, it is clear that the resistors dissipating most of the power have the largest impact on the total equivalent resistance since, by construction, the system can be described as branches of serial resistances placed in a parallel arrangement. In other words, the hot-spots seem to be accountable for most of the variations in the measured resistance. In more advanced studies, it would be of much interest to introduce singularities in the model, such as structural defects, which could also contribute to the emergence of localized hot-spots.

\section{Localized film modification}

To support this conclusion, the CNFs film was selectively damaged, to identify the role of the different temperature regions on the overall resistance. Figure 4a shows the record of the resistance of a CNFs film and the temperature evolution at a selected hot-spot ( $\psi$ region, see Figure $4 \mathrm{~b}$ ) during three self-heating pulses on (I) the original film; (II) after mechanically scratching a cold region $(\kappa$, see Figure $4 \mathrm{~b}$ ) and (III) after scratching the hot-spot $\psi$. Comparing (I) and (II), before and after modifying a cold region, the observed change on the resistance values and on the temperature profile are very small. Also, the temperature at the hot-spot $\psi$ shows essentially the same transient behavior: a fast process $A$ followed by a slower process B, which were characteristic of a hot-spot. In contrast, at (III) (i.e., after destroying a hot-spot) the resistance value changed dramatically and the thermalization only displayed the slower trend (Process B).

\section{Functional effects of localized self-heating}

These results are consistent with the behavior predicted by the model, proving that the resistance measurements are extremely sensitive to the hot-spot properties. Therefore, self-heating calibration methods based on monitoring the electrical resistance value are mainly calibrating the hot-spots, and not the mean film 
temperature, as assumed in previous works ${ }^{22,26,34}$. Conversely, this also means that the electrical resistance mostly reflects changes in the hot-spots. For example, in the case of interactions with gases, which are thermally activated process used in gas sensing, the electrical resistance variations would essentially account for the interactions occurring at the hot-spots. Equally, this understanding can help to prevent the interference of local heating in quantum transport experiments in highly confined materials. Concerning power consumption, the fact that the heating effect (and their electrical consequences) concentrate in specific (small) regions, explains why self-heating in films of nanowires is comparable in efficiency with self-heating in individual nanowires. The same argument applies for the comparable response dynamics recently observed. Finally, the fact that a large random network of nanoresistors exhibit hot-spots, mainly corresponding to high resistance regions with proper connectivity, suggests that this functionality emerges from the self-organization of the system. For a given nanowire distribution, a set of hot-spots is defined, and their temperature can be calibrated and monitored just tracking the macroscopic resistance. (a)


Agreement n. 336917. J.D. Prades acknowledges the support of the Serra Húnter Programme. A. Cirera acknowledges support from ICREA Academia program and from BBVA foundation.

\section{Notes and references}

1

F. Hernandez-Ramirez, A. Tarancon, O. Casals, E. Pellicer, J. Rodriguez, A. Romano-Rodriguez, J. Morante, S. Barth and S. Mathur, Phys. Rev. B, 2007, 76, 085429. L. F. Zhu, J. C. She, J. Y. Luo, S. Z. Deng, J. Chen, X. W. Ji and N. S. Xu, Sensors Actuators B Chem., 2011, 153, 354-360. N. D. Chinh, N. Van Toan, V. Van Quang, N. Van Duy, N. D. Hoa and N. Van Hieu, Sensors Actuators B Chem., 2014, 201, 7-12.

D. Zhang, J. Tong and B. Xia, Sensors Actuators B Chem., 2014, 197, 66-72.

5 C. Y. Jin, Z. Li, R. S. Williams, K. C. Lee and I. Park, Nano Lett., 2011, 11, 4818-4825.

J.-H. Ahn, J. Yun, D.-I. Moon, Y.-K. Choi and I. Park, Nanotechnology, 2015, 26, 095501.

See

http://www.keithley.com/events/semconfs/webseminars/n anoseminar3/for 'How to Avoid Self-Heating Effects on Nanoscale Devices' (last accessed September 1, 2015)., . D. Vasileska, K. Raleva and S. Goodnick, in Cutting Edge Nanotechnology, 2010, pp. 33-61.

9 O. Semenov, A. Vassighi and M. Sachdev, IEEE Trans. Device Mater. Reliab., 2006, 6, 17-27.

10 E. Pop, D. Mann, Q. Wang, K. Goodson and H. Dai, Nano Lett., 2006, 6, 96-100. B. Kumar, K. Min, M. Bashirzadeh, a B. Farimani, M. H Bae, D. Estrada, Y. D. Kim, P. Yasaei, Y. D. Park, E. Pop, N. R. Aluru and a Salehi-Khojin, Nano Lett., 2013, 13, 19621968.

Figure 4. (a) Electrical resistance of the CNFs film and temperature at region $\psi$ (see images in (b)) recorded during three self-heating processes. Along the experiments, the CNFs film was mechanically scratched: first the region $\kappa$ (an area without hot-spots) and then, the region $\psi$ (an area with hot-spots). (b) Thermographic images acquired at the steady state of each one of the three self-heating pulses.

\section{Conclusions}

Temperature distribution in a self-heated random network on nanostructures in self-heating operation is highly inhomogeneous and heat concentrates in certain hot-spots. Such highly localized heating could explain the high power efficiencies observed in relatively large systems, provided that they were not compact and homogeneous but formed by a fine mesh of e.g. 1D nanostructures. This understanding opens a path to design highly efficient self-heating systems, based on random or pseudo-random distributions of 1D nanowires.

\section{Acknowledgements}

The research leading to these results has received funding from the European Research Council under the European Union's Seventh Framework Programme (FP/2007-2013) / ERC Grant
A. Salehi-khojin, K. Y. Lin, C. R. Field and R. I. Masel, Science (80-. )., 2010, 329, 1327-1331.

13 A. Salehi-Khojin, F. Khalili-Araghi, M. a. Kuroda, K. Y. Lin, J.P. Leburton and R. I. Masel, ACS Nano, 2010, 5, 153-158. F. Hernandez-Ramirez, J. D. Prades, a Tarancon, S. Barth, O. Casals, R. Jiménez-Diaz, E. Pellicer, J. Rodriguez, M. a Juli, a Romano-Rodriguez, J. R. Morante, S. Mathur, a Helwig, J. Spannhake and G. Mueller, Nanotechnology, 2007, 18, 495501.

J. D. Prades, R. Jimenez-Diaz, F. Hernandez-Ramirez, S. Barth, A. Cirera, A. Romano-Rodriguez, S. Mathur and J. R. Morante, Appl. Phys. Lett., 2008, 93, 123110.

E. Strelcov, S. Dmitriev, B. Button, J. Cothren, V. Sysoev and

J. D. Prades, R. Jimenez-Diaz, F. Hernandez-Ramirez, a. Cirera, a. Romano-Rodriguez and J. R. Morante, Sensors Actuators, B Chem., 2010, 144, 1-5.

J. D. Prades, R. Jimenez-Diaz, F. Hernandez-Ramirez, J. Pan, A. Romano-Rodriguez, S. Mathur and J. R. Morante, Appl. Phys. Lett., 2009, 95, 053101.

J. D. Prades, F. Hernández-Ramírez, T. Fischer, M. Hoffmann, R. Müller, N. López, S. Mathur and J. R. Morante, Appl. Phys. Lett., 2010, 97, 243105. F. Hernandez-Ramirez, J. D. Prades, R. Jimenez-Diaz, T. Fischer, A. Romano-Rodriguez, S. Mathur and J. R. Morante, Phys. Chem. Chem. Phys., 2009, 11, 7105-7110. M. Afshar, E. M. Preiß, T. Sauerwald, M. Rodner, D. Feili, M. Straub, K. König, A. Schütze and H. Seidel, Sensors A. Kolmakov, Nanotechnology, 2008, 19, 355502. 
Actuators B Chem., 2015, 215, 525-535.

O. Monereo, J. D. Prades and a. Cirera, Sensors Actuators B Chem., 2015, 211, 489-497.

23 J. Guilera, C. Fàbrega, O. Casals, F. Hernández-Ramírez, S. Wang, S. Mathur, F. Udrea, A. De Luca, S. Z. Ali, A. RomanoRodríguez, J. D. Prades and J. R. Morante, Sensors Actuators B Chem., 2015, 221, 104-112.

24 V. Jelicic, M. Magno, K. Chikkadi, C. Roman, C. Hierold, V. Bilas and L. Benini, in Workshop on Advances in Sensors and Interfaces (IWASI), 2015 6th IEEE International, IEEE, Gallipoli, 2015, pp. 271-276.

25 O. Monereo, J. D. Prades and A. Cirera, Sensors Actuators $B$ Chem., 2015, 211, 489-497.

26 O. Monereo, O. Casals, J. D. Prades and A. Cirera, Sensors Actuators B Chem., 2015, In press.

27 J. D. Prades, R. Jimenez-Diaz, F. Hernandez-Ramirez, L. Fernandez-Romero, T. Andreu, A. Cirera, A. RomanoRodriguez, A. Cornet, J. R. Morante, S. Barth and S. Mathur, J. Phys. Chem. C, 2008, 112, 14639-14644.

J. Vera-Agullo, H. Varela-Rizo, J. A. Conesa, C. Almansa, C. Merino and I. Martin-Gullon, Carbon N. Y., 2007, 45, 27512758.

29 M. Schmidt, A. Schütze and S. Seelecke, Int. J. Refrig., 2015, 54, 88-97.

30 M. Schmidt, J. Ullrich, A. Wieczorek, J. Frenzel, A. Schütze, G. Eggeler and S. Seelecke, Shape Mem. Superelasticity, 2015, 132-141.

31 J. D. Prades, R. Jimenez-Diaz, F. Hernandez-Ramirez, a. Cirera, a. Romano-Rodriguez and J. R. Morante, Sensors Actuators B Chem., 2010, 144, 1-5.

32 J. D. Prades, R. Jimenez-Diaz, F. Hernandez-Ramirez, J. Pan, a. Romano-Rodriguez, S. Mathur and J. R. Morante, Appl. Phys. Lett., 2009, 95, 93-96.

33 J. D. Prades, F. Hernández-Ramírez, T. Fischer, M. Hoffmann, R. Müller, N. López, S. Mathur and J. R. Morante, Appl. Phys. Lett., 2010, 97, 243105.

34 J. D. Prades, R. Jimenez-Diaz, F. Hernandez-Ramirez, S. Barth, J. Pan, A. Cirera, A. Romano-Rodriguez, S. Mathur and J. R. Morante, Int. J. Nanotechnol., 2009, 6, 860. Y. Yue, J. Zhang and X. Wang, Small, 2011, 7, 3324-3333.

36 Y. Yue, G. Eres, X. Wang and L. Guo, Appl. Phys. A Mater. Sci. Process., 2009, 97, 19-23.

37 I. Calizo, A. A. Balandin, W. Bao, F. Miao and C. N. Lau, Nano Lett., 2007, 7, 2645-2649.

38 S. Claramunt, O. Monereo, M. Boix, R. Leghrib, J. D. Prades, a. Cornet, P. Merino, C. Merino and a. Cirera, Sensors Actuators, B Chem., 2013, 187, 401-406.

39 N. Chiodarelli1, S. Masahito, Y. Kashiwagi, Y. Li, K. Arstila, O. Richard, D. J. Cott, M. Heyns, S. De Gendt, G.

Groeseneken and P. M. Vereecken, Nanotechnology, 2011, 22, 085302. 